\title{
A systematic approach for testing expression of human full-length proteins in cell-free expression systems Claudia Langlais ${ }^{\dagger 1}$, Birgit Guilleaume ${ }^{\dagger 2}$, Nadja Wermke ${ }^{2}$, Tina Scheuermann², Lars Ebert ${ }^{2}$, Joshua LaBaer ${ }^{3}$ and Bernhard Korn*4
}

\author{
Address: ${ }^{1}$ MRC Toxicology Unit, Protein Profiling Group, Hodgkin Building, Lancaster Road, Leicester, LE1 9HN, UK, ${ }^{2}$ German Ressource Center, \\ Im Neuenheimer Feld 515, D-69120 Heidelberg, Germany, ${ }^{3}$ Institute of Proteomics, Harvard Medical School, 240 Longwood Avenue, Boston, MA \\ 02129, USA and ${ }^{4}$ German Cancer Research Center, Genomics \& Proteomics Core Facilities, Im Neuenheimer Feld 515, D-69120 Heidelberg, \\ Germany \\ Email: Claudia Langlais -cl40@leicester.ac.uk; Birgit Guilleaume - bguilleaume@web.de; Nadja Wermke - n.wermke@dkfz.de; \\ Tina Scheuermann - t.scheuermann@dkfz.de; Lars Ebert - l.ebert@dkfz.de; Joshua LaBaer - josh@hms.harvard.edu; \\ Bernhard Korn* - b.korn@dkfz.de \\ * Corresponding author †Equal contributors
}

Published: 3 October 2007

BMC Biotechnology 2007, 7:64 doi:10.1 I86/1472-6750-7-64

This article is available from: http://www.biomedcentral.com/l472-6750/7/64

(C) 2007 Langlais et al.; licensee BioMed Central Ltd.

This is an Open Access article distributed under the terms of the Creative Commons Attribution License (http://creativecommons.org/licenses/by/2.0), which permits unrestricted use, distribution, and reproduction in any medium, provided the original work is properly cited.
Received: 23 April 2007

Accepted: 3 October 2007

\begin{abstract}
Background: The growing field of proteomics and systems biology is resulting in an ever increasing demand for purified recombinant proteins for structural and functional studies. Here, we show a systematic approach to successfully express a full-length protein of interest by using cellfree and cell-based expression systems.

Results: In a pre-screen, we evaluated the expression of 960 human full-length open reading frames in Escherichia coli (in vivo and in vitro). After analysing the protein expression rate and solubility, we chose a subset of 87 plasmids yielding no protein product in $E$. coli in vivo. These targets were subjected to a more detailed analysis comparing a prokaryotic cell-free $E$. coli system with an eukaryotic wheat germ system. In addition, we determined the expression rate, yield and solubility of those proteins. After sequence optimisation for the $E$. coli in vitro system and generating linear templates for wheat germ expression, the success rate of cell-free protein expression reached $93 \%$.

Conclusion: We have demonstrated that protein expression in cell-free systems is an appropriate technology for the successful expression of soluble full-length proteins. In our study, wheat germ expression using a two compartment system is the method of choice as it shows high solubility and high protein yield.
\end{abstract}

\section{Background}

With the sequencing of the human genome completed and with mRNA/cDNA identification rapidly progressing, many potential novel genes have been discovered and attention has turned to the function and structure of the predicted proteins [1-4]. In order to study these novel gene products, sufficient amounts of protein generally obtained through recombinant protein expression are required. The (high-throughput) expression and characterisation of unknown and poorly characterised human proteins is a main objective of recombinant proteomic studies today. 
Escherichia coli is the most commonly used prokaryotic expression system for the high-level production of recombinant proteins in vivo [5] and has already been used successfully in high-throughput protein expression and purification studies $[4,6]$. The use of E. coli has many advantages, including the ease of growth and manipulation of the organism and the availability of many different vectors and host strains that have been developed over the years. However, the use of $E$. coli also has limitations, such as the aggregation of protein in insoluble inclusion bodies, problems with the expression of gene products toxic to the physiology of the host cell or proteolytic degradation of proteins in the cytoplasm [7]. In light of these difficulties, cell-free expression systems are becoming increasingly popular [8-14]. The in vitro systems have several advantages, including rapid protein synthesis [15], the possibility to express toxic gene products [16] and constructs that otherwise would be proteolytically degraded. Furthermore, it is possible to express proteins with up to 10 putative transmembrane domains as reported recently [17]. The compatibility with PCR-generated templates as well as plasmids allows the in vitro expression reaction with $E$. coli extract to be optimised using silent mutations within PCR products [18]. These sequence optimisations reduce unfavourable secondary structures in mRNA and thus improve the success rate of translation and protein expression. In contrast, for cellfree protein expression with wheat germ lysate sequence optimisation is not necessary because of the eukaryotic nature of this source.

For protein expression analyses, a comprehensive cDNA collection is available at the German Ressource Center (RZPD). The full-length open reading frames (ORFs) are cloned into an entry vector by utilizing the Gateway ${ }^{\otimes}$ cloning technology (Invitrogen). Untranslated regions are excluded and only the open reading frame is cloned into the selected vector, either with or without a stop codon. For protein expression, the open reading frame can be moved into any desired expression vector by homologous recombination. Thus, a protein can be expressed with or without a tag and the tag itself can easily be selected and altered by choosing the appropriate destination vector.

The aim of this study was to evaluate alternatives to protein expression in E.coli in vivo especially for those ORFs yielding no protein in this system. Therefore we investigated protein expression in two different in vitro systems: E. coli and wheat germ extract. The performance of these systems was analysed and optimised in respect to expression rate, protein yield and solubility. Altogether, we tested the expression of 960 human full-length proteins in vivo and in vitro using standardised conditions.

\section{Results \\ Comparison of in vivo and in vitro Escherichia coli expressions}

We used 960 randomly selected fully sequence-verified human open reading frames with a broad range of expected molecular weights (from less than $8 \mathrm{kDa}$ up to $134 \mathrm{kDa}$, average of $35 \mathrm{kDa}$ ), different predicted subcellular localisations and biochemical functions including membrane proteins. The ORFs were cloned into an expression vector (pDEST17-D18), for production of proteins with an $\mathrm{N}$-terminal 6xHis-tag. Identical constructs were used for protein expression in vitro and also for transformation of bacteria and expression in vivo. Protein expression was analysed by western blotting using an antiHis antibody.

In E. coli in vivo 629 out of 960 proteins, and in vitro 456 out of 960 proteins were successfully expressed. Protein expression in bacteria was unsuccessful either because clones were generated, which did not show protein expression (233 samples) or the transformation failed completely (98 samples). Considering the overlap of both expression systems, 206 full open reading frames yielded no protein product in vivo andin vitro. In contrast, 331 targets were expressed in vivo andin vitro with an average molecular weight of $33 \mathrm{kDa}$. Among these, 57 clones showed an expression rate of 4,86 clones of 3 and 7 clones of 2. Furthermore, 424 targets expressed in either system with an average moleclar weight of $32 \mathrm{kDa}$.

\section{Optimisation of $\mathrm{E}$. coli expression in vitro}

We next examined the effect of sequence optimisation on protein expression rate and protein yield by selecting randomly 87 out of the 960 ORFs (Figure 1, Table 1) where protein expression had been unsuccessful in vivo or where transformation had failed in BL21(DE3)pLysS. Three kinds of linear PCR templates were generated (Figure 2): (i) a C-terminal wild type with C-terminal $6 x$ His-tag (ii) a C-terminal mutant with C-terminal 6xHis-tag and inserted silent mutation at the $\mathrm{N}$-terminus (iii) a $\mathrm{N}$-terminal wild type with N-terminal 6xHis-tag and no attachment sites (att-sites); For the C- and N-terminal wild type template the ORF is identical to the original ORF in the plasmid DNA.

\section{Influence of sequence optimisation on protein expression rate}

Of these 87 samples, 37 samples (43\%) were successfully expressed in vitro using the original plasmid DNA. After sequence optimisation, we increased the success rate of protein expression up to 74 samples (85\%) in the cell-free E. coli system (Figure 3, 4).

When analysing the results of those samples which had previously not shown expression in vitro (50 samples), we 
Table I: Subset of 87 clones tested in E. coli in vitro and in wheat germ expression. ORF Nr.: Clone identifier; RZPD Clone ID: Available clones at RZPD GmbH; Hit Acc. Nr.: Best BLAST hit of DNA sequence. Molecular weight was calculated by translation of the DNA sequence. Expression/solubility were assigned values from 0 (no expression/no protein detectable in the supernatant) to 4 (very strong band/protein band in the supernatant is stronger than in the pellet). The column 'yielded by' is indicated as follows: WG C: wheat germ C-terminal, WG: wheat germ, C- and N-terminal, WG N: wheat germ, N-terminal, RTS CW: E. coli in vitro C-terminal wildtype, RTS CM: E. coli in vitro C-terminal mutant, RTS NW: E. coli in vitro N-terminal wildtype, RTS PI: E. coli in vitro original plasmid.

\begin{tabular}{|c|c|c|c|c|c|c|c|}
\hline ORF Nr & RZPDCloneID & Hit Acc No & Gene symbol & Mw in $\mathbf{k D a}$ & Best expr. rate & Yielded by & Best solubility \\
\hline 264 & RZPDo834B052 & NM_00I677 & ATPIBI & 36 & 4 & RTS NW & 3 \\
\hline 400 & RZPDo834D022 & NM_002573 & PAFAHIB3 & 27 & 4 & WG, RTS CM NW & 4 \\
\hline 433 & & NM_000318 & PXMP3 & 32 & 4 & WG, RTS CW CM NW & 4 \\
\hline 464 & RZPDo834F0I2 & NM_006793 & PRDX3 & 29 & 4 & WG C, RTS CM & 4 \\
\hline 505 & & NM_003I87 & TAF9 & 30 & 4 & WG & 3 \\
\hline 531 & & NM_0I 2222 & MUTYH & 23 & 4 & WG & 3 \\
\hline 562 & RZPDo834B043 & NM_000075 & CDK4 & 35 & 4 & RTS CM NW PI & 3 \\
\hline 571 & RZPDo834B083 & NM_003 I 82 & $\mathrm{TACl}$ & 13 & 4 & WG C, RTS CW CM & 4 \\
\hline 616 & RZPDo834D033 & NM_000550 & TYRPI & 61 & 3 & RTS PI & 2 \\
\hline 636 & & NM_000612 & IGF2 & 22 & 4 & WG C, RTS CW CM & 4 \\
\hline 637 & RZPDo834E0I3 & NM_0I5646 & RAPIB & 22 & 4 & WG, RTS CW CM NW & 4 \\
\hline 639 & & NM_0025I2 & NME2 & 14 & 4 & WG N, RTS NW & 3 \\
\hline 690 & RZPDo834FI 23 & NM_006923 & SDF2 & 23 & 4 & WG C, RTS CW CM & 4 \\
\hline 694 & RZPDo834G023 & NM_020470 & YIFI & 32 & 4 & WG $N$ & 3 \\
\hline 728 & RZPDo834E05II & NM_000434 & NEUI & 46 & 4 & RTS NW & 2 \\
\hline 741 & RZPDo834H073 & NM_002799 & PSMB7 & 30 & 4 & WG C & 4 \\
\hline 772 & RZPDo834C03II & NM_002804 & PSMC3 & 46 & 4 & WG N, RTS CM & 4 \\
\hline 777 & RZPDo834C04II & NM_003908 & EIF2S2 & 39 & 4 & WG & 4 \\
\hline 831 & & NM_004394 & DAP & 12 & 3 & WG & 4 \\
\hline 832 & & NM_002966 & SIOOAIO & 12 & 4 & WG, RTS CM NW & 4 \\
\hline 833 & RZPDo834AI 24 & NM_0I7503 & SURF2 & 30 & 4 & WG C & 4 \\
\hline 840 & RZPDo834B024 & NM_005499 & UBA2 & 72 & 3 & WG C & 4 \\
\hline 842 & & NM_005942 & MOCSI & 24 & 4 & WG N, RTS CM & 3 \\
\hline 855 & & NM_002I34 & HMOX2 & 36 & 4 & RTS NW & 4 \\
\hline 861 & RZPDo834C034 & NM_006370 & VTIIB & 27 & 4 & WG N, RTS NW PI & 3 \\
\hline 868 & RZPDo834C084 & NM_005892 & FMNLI & 53 & 4 & WG, RTS CM NW & 3 \\
\hline 873 & RZPDo834CI04 & NM_007363 & NONO & 55 & 4 & WG C & 3 \\
\hline 881 & & NM_002622 & PFDNI & 15 & 4 & WG C & 4 \\
\hline 898 & RZPDo834DII4 & NM_006II7 & $\mathrm{PECl}$ & 21 & 4 & WG N, RTS CW & 4 \\
\hline 901 & RZPDo834E024 & NM_03I 263 & HNRPK & 51 & 4 & RTS CW NW & 4 \\
\hline 904 & & NM_00440I & DFFA & 13 & 4 & WG N & 4 \\
\hline 906 & & NM_006693 & CPSF4 & 31 & 4 & WG C, RTS CM & 4 \\
\hline 915 & RZPDo834F024 & M55654 & TBP & 38 & 4 & WG N, RTS NW PI & 3 \\
\hline 918 & & NM_004I84 & WARS & 54 & 3 & WG N & 4 \\
\hline 921 & RZPDo834F064 & NM_004309 & ARHGDIA & 23 & 4 & RTS CM NW & 3 \\
\hline 924 & RZPDo834F094 & NM_00286I & PCYT2 & 44 & 4 & WG C & 4 \\
\hline 930 & RZPDo834G0I4 & NM_00I55I & IGBPI & 40 & 4 & WG, RTS PI & 4 \\
\hline 932 & RZPDo834G034 & NM_002070 & GNAI2 & 41 & 4 & WG C, RTS NW PI & 4 \\
\hline 935 & & NM_00I0I4835 & PAK4 & 64 & 2 & WG C & 3 \\
\hline 936 & RZPDo834G064 & NM_002074 & GNBI & 38 & 3 & RTS NW & 3 \\
\hline 939 & RZPDo834G074 & NM_00632I & $\mathrm{ARIH} 2$ & 58 & 3 & WG C, RTS PI & 4 \\
\hline 940 & RZPDo834G084 & NM_0I3296 & GPSM2 & 55 & 2 & WG C & 4 \\
\hline 943 & RZPDo834GII4 & NM_001863 & COX6BI & 11 & 4 & WG, RTS CW CM PI & 4 \\
\hline
\end{tabular}


Table I: Subset of 87 clones tested in E. coli in vitro and in wheat germ expression. ORF Nr.: Clone identifier; RZPD Clone ID: Available clones at RZPD GmbH; Hit Acc. Nr.: Best BLAST hit of DNA sequence. Molecular weight was calculated by translation of the DNA sequence. Expression/solubility were assigned values from 0 (no expression/no protein detectable in the supernatant) to 4 (very strong band/protein band in the supernatant is stronger than in the pellet). The column 'yielded by' is indicated as follows: WG C: wheat germ C-terminal, WG: wheat germ, C- and N-terminal, WG N: wheat germ, $\mathbf{N}$-terminal, RTS CW: $E$. coli in vitro C-terminal wildtype, RTS CM: E. coli in vitro C-terminal mutant, RTS NW: E. coli in vitro $\mathbf{N}$-terminal wildtype, RTS PI: E. coli in vitro original plasmid. (Continued)

\begin{tabular}{|c|c|c|c|c|c|c|c|}
\hline 944 & & NM_004537 & NAPILI & 46 & 4 & WG & 4 \\
\hline 945 & RZPDo834H0I4 & NM_152925 & RBMI2 & 59 & 4 & WG C & 4 \\
\hline 947 & RZPDo834H034 & NM_001017957 & OS-9 & 70 & 4 & WG C & 4 \\
\hline 1033 & & NM_0073I7 & KIF22 & 74 & 0 & & 0 \\
\hline 1068 & & NM_206900 & RTN2 & 52 & 4 & WG C & 4 \\
\hline 1082 & & NM_0I8074 & FLJI0374 & 37 & 4 & WG; RTS CW CM & 4 \\
\hline 1091 & & NM_00I5I2 & GSTA4 & 26 & 4 & WG & 4 \\
\hline 1093 & RZPDo834A0I5 & NM_00I647 & APOD & 22 & 4 & WG C, RTS CM & 4 \\
\hline 1101 & & NM_001643 & APOA2 & 12 & 4 & WG N & 3 \\
\hline 1115 & & NM_00726I & CMRF-35H & 25 & 4 & WG, RTS CM NW & 4 \\
\hline 1189 & RZPDo834HI06 & NM_00I425 & EMP3 & 19 & 4 & WG & 4 \\
\hline 1294 & RZPDo834A035 & NM_0I4876 & KIAA0063 & 24 & 4 & WG & 4 \\
\hline 1330 & & NM_0028I6 & PSMDI 2 & 53 & 4 & WG C & 4 \\
\hline 1453 & RZPDo834BII5 & NM_198216 & SNRPB & 25 & 4 & WG C, RTS CM & 3 \\
\hline 1454 & RZPDo834H07II & NM_00684I & SLC38A3 & 56 & 4 & WG, RTS NW & 3 \\
\hline $146 \mid$ & RZPDo834C025 & NM_004047 & ATP6V0B & 22 & 3 & WG C & I \\
\hline 1462 & RZPDo834C035 & NM_003I45 & SSR2 & 21 & 4 & WG C, RTS CM & 3 \\
\hline 1480 & & NM_000984 & RPL23A & 18 & 3 & WG & 4 \\
\hline 1485 & RZPDo834D025 & NM_0I4860 & SUPT7L & 47 & 4 & WG & 4 \\
\hline 1487 & RZPDo834D035 & NM_198I20 & EBAG9 & 25 & 4 & WG C & 3 \\
\hline 1533 & RZPDo834F075 & NM_0I3300 & HSU79274 & 31 & 4 & WG C & 3 \\
\hline 1554 & RZPDo834G095 & NM_00I778 & CD48 & 28 & 4 & RTS NW & 3 \\
\hline 1555 & RZPDo834GI05 & NM_0191II & HLA-DRA & 29 & 4 & RTS CM & 3 \\
\hline 1575 & RZPDo834H085 & NM_004233 & CD83 & 23 & 4 & WG, RTS CM & 4 \\
\hline 1576 & RZPDo834H095 & NM_007024 & PL6 & 39 & 2 & WG N & I \\
\hline 1642 & & NM_003490 & SYN3 & 64 & 2 & WG & 4 \\
\hline 1670 & & NM_00684I & SLC38A3 & 56 & 4 & WG N, RTS NW & 3 \\
\hline 1734 & RZPDo834F05II & NM_00I436 & FBL & 34 & 4 & WG C & 4 \\
\hline 1736 & RZPDo834G05 I I & NM_004343 & CALR & 49 & 4 & WG C & 4 \\
\hline 2066 & & NM_0I5723 & PNPLA8 & 89 & 0 & & 0 \\
\hline 2225 & RZPDo834H022I & NM_018I27 & ELAC2 & 93 & 0 & & 0 \\
\hline 2229 & RZPDo834A046 & NM_005I3 & REC8LI & 63 & 3 & WG C, RTS CM & 4 \\
\hline 2504 & & NM_199053 & FLJ 12716 & 65 & 4 & RTS CW CM NW & 3 \\
\hline 2724 & & NM_I73|57 & NR4AI & 65 & 4 & WG N & 2 \\
\hline 2871 & & NM_018099 & MLSTDI & 60 & 4 & RTS CW CM NW & 3 \\
\hline 2938 & RZPDo834H042I & NM_0I5072 & TTLL5 & 92 & 0 & & 0 \\
\hline 2949 & RZPDo834EII2I & NM_020748 & KIAAI 287 & 135 & 0 & & 0 \\
\hline 2959 & & NM_021932 & RIC8 & 17 & 4 & WG, RTS PI & 4 \\
\hline 2962 & RZPDo834H062I & NM_014I49 & HSPC049 & 78 & 2 & RTS CM NW & 0 \\
\hline 2964 & RZPDo834FII2I & NM_003263 & TLRI & 91 & 4 & RTS CM NW & 4 \\
\hline 2968 & RZPDo834G082I & NM_00I040428 & SPATA7 & 65 & 4 & RTS CW CM NW & 3 \\
\hline 2973 & & NM_032292 & FLJ20203 & 91 & 4 & RTS CW CM NW & 3 \\
\hline 2978 & RZPDo834H072I & NM_0I4585 & SLC40AI & 63 & 0 & & 0 \\
\hline 2979 & RZPDo834E082 I & NM_0I3277 & RACGAPI & 71 & 4 & RTS CM & 0 \\
\hline
\end{tabular}




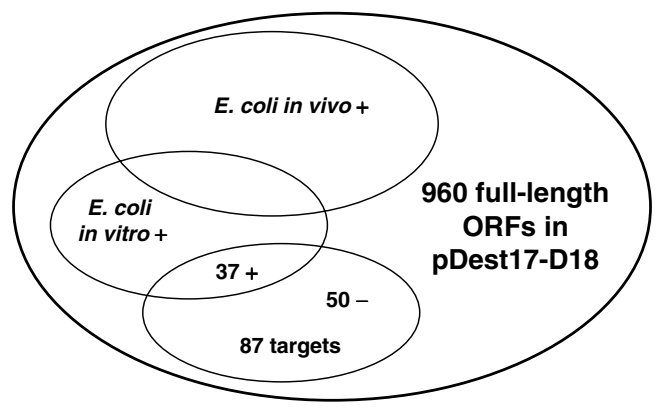

87 targets (37 E. coli in vitro $+/ 50$ E. coli in vitro and in vivo-)

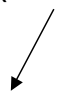

E. coli expression in vitro using optimised linear PCR products $(74+/ 13-)$

\section{Figure I}

Scheme of the experimental strategy. Successful protein expression is indicated by + , unsuccessful protein expression by -.

found that following sequence optimisation 37 (74\%) proteins were expressed.

\section{Influence of sequence optimisation on protein yield}

To assess the protein expression yield of PCR products after sequence optimisation, we evaluated 37 samples that

(i) C-terminal wild type

\begin{tabular}{l|l|l} 
att1 & ORF & att2
\end{tabular}

(ii) C-terminal mutant

$\begin{array}{lll}\text { att1 } & \text { ORF }\end{array}$

(ii) N-terminal wild type

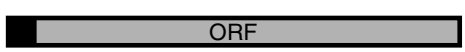

空究 silent mutation

6xHis-tag

\section{Figure 2}

Optimised linear templates for $E$. coli in vitro expression. Three kinds of linear PCR-products were generated to investigate the effect on protein expression rate and yield. (i) Cterminal wild type with $\mathrm{C}$-terminal $6 \times \mathrm{H}$ is-tag (ii) $\mathrm{C}$-terminal mutant with $\mathrm{C}$-terminal $6 \mathrm{xH}$ is-tag and inserted silent mutation at the $\mathrm{N}$-terminus (iii) $\mathrm{N}$-terminal wild type with $\mathrm{N}$-terminal 6xHis-tag and no attachment sites (att-sites); For the $\mathrm{C}$ - and $\mathrm{N}$-terminal wild type template the ORF is identical to the original ORF in the plasmid DNA.

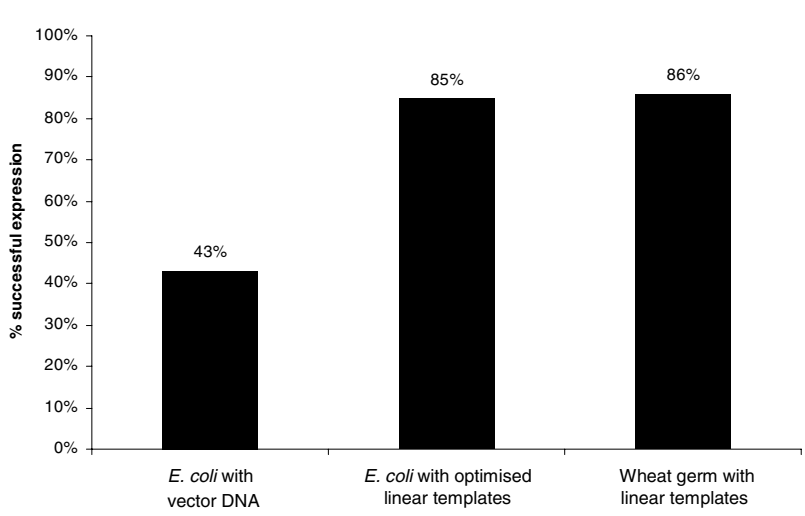

Figure 3

Comparison of in vitro expression of 87 targets in $E$. coli and wheat germ.

had previously expressed protein in vitro from original plasmid DNA. Protein yield was determined by analysis of protein bands on western blots. Bands were given marks from 0 (no expression) to 4 (very strong). Here, we discovered that $65 \%$ of expressions (24 samples) showed an improvement in the protein yield compared to expressions using original plasmid DNA and another 19\% showed similar protein yields. A smaller amount of protein was expressed in only 6 cases $(16 \%)$ using the optimised PCR products. Among these were 3 samples which did not express protein at all. In summary, after analysis of 87 expressions in vitro with optimised PCR-products, 16 samples (18\%) revealed no protein product in vivo or in vitro in the $E$. coli systems (Table 2 ).

\section{Wheat germ expression in vitro}

The aim of this experiment was to elucidate whether the wheat germ system would show an increase in the success rate and protein yield of the 87 selected open reading frames compared to the optimised in vitro expressions in E. coli. Two wild type PCR constructs were made for each open reading frame, one for production of a protein with a C-terminal 6xHis-tag and another for a protein with a $\mathrm{N}$ terminal 6xHis-tag (Figure 5). A total of 75 proteins could be expressed in wheat germ lysate with either a C- or a $\mathrm{N}$ terminal 6xHis-tag (86\%, Figure 3). Out of the 16 open reading frames which were not expressed in the $E$. coli systems, 10 were now successfully expressed using wheat germ lysate (Table 2). However, 6 open reading frames did not express in the wheat germ system, but were previously successfully expressed in vitro in E. coli (Table 2). On average, based on western blotting analyses, the protein yield was higher in the wheat germ compared to expressions in the E. coli in vitro system, for identical human ORFs. 


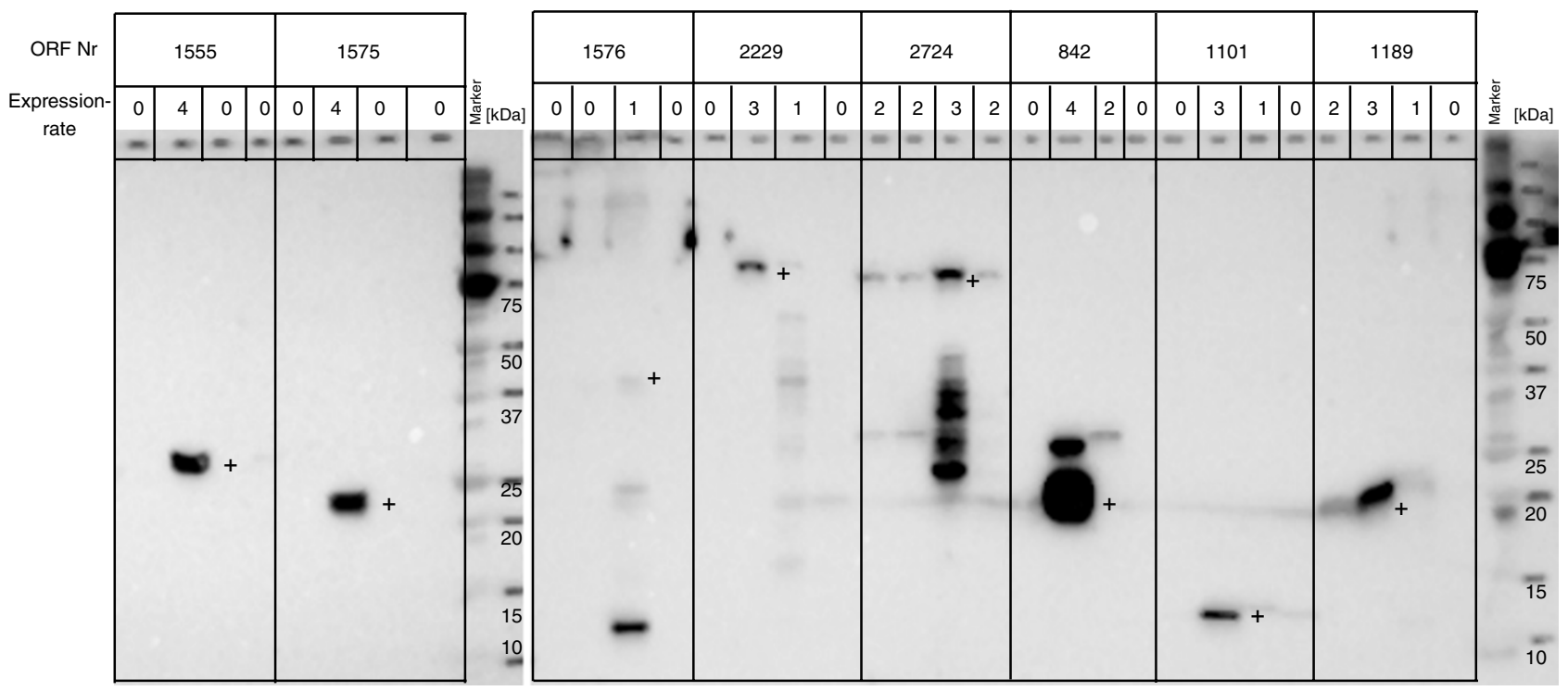

\section{Figure 4}

E.coli expression in vitro. Presented are western blots of 8 targets expressed with C-terminal wild type, C-terminal mutant, $\mathrm{N}$ terminal wild type and original plasmid DNA templates (from left to right). Successful protein expression was defined for values $2-4$ and unsuccessful protein expression for values of 0 and I. Bands of the expected size are marked with a + .

Table 2: Proteins not expressing in E. coli in vitro or in wheat germ or in both systems. Molecular weight was calculated by translation of the DNA sequence. Localization information was taken from the Uniprot database. Domain information was retrieved from the Pfam database: cc: coiled coil, tms: transmembrane segment, sp signal peptide. Empty fields correspond to no assignment in the database.

\begin{tabular}{|c|c|c|c|c|c|c|}
\hline Hit Acc. No. & Gene symbol & $M w$ in $\mathrm{kDa}$ & Localization & Domains & Expression in E. coli in vitro & Expression in wheat germ \\
\hline NM_0073I7 & KIF22 & 74 & nuclear & Icc, & no & no \\
\hline NM_015723 & PNPLA8 & 89 & membrane & & no & no \\
\hline NM_020748 & KIAAI 287 & 135 & $\mathrm{n} / \mathrm{a}$ & & no & no \\
\hline NM_018I27 & ELAC2 & 93 & nuclear & & no & no \\
\hline NM_015072 & TTLL5 & 92 & $\mathrm{n} / \mathrm{a}$ & $3 c c$, & no & no \\
\hline NM_0I4585 & SLC40AI & 63 & membrane & „10tms & no & no \\
\hline NM_020470 & YIFIA & 32 & membrane &, $5 \mathrm{tms}$ & no & yes \\
\hline NM_004394 & DAP & 12 & secreted & & no & yes \\
\hline NM_001014835 & PAK4 & 64 & $\mathrm{n} / \mathrm{a}$ & & no & yes \\
\hline NM_0I3296 & GPSM2 & 55 & $\mathrm{n} / \mathrm{a}$ & & no & yes \\
\hline NM_006812 & OS-9 & 70 & $\mathrm{n} / \mathrm{a}$ & Icclspltms & no & yes \\
\hline NM_0I4860 & SUPT7L & 47 & $\mathrm{n} / \mathrm{a}$ & Icc, & no & yes \\
\hline NM_003908 & EIF2S2 & 39 & nuclear & & no & yes \\
\hline NM_00632I & $\mathrm{ARIH} 2$ & 58 & nucleus & $2 c c$, & no & yes \\
\hline NM_002816 & PSMDI 2 & 53 & cytosol & Icc, & no & yes \\
\hline NM_000984 & RPL23A & 18 & cytosol & & no & yes \\
\hline NM_002I 34 & $\mathrm{HMOX} 2$ & 36 & microsomal & Icc, I tms & yes & no \\
\hline NM_018099 & MLSTDI & 60 & intracellular &, $2 \mathrm{tms}$ & yes & no \\
\hline NM_013277 & RACGAPI & 71 & intracellular & Icc, & yes & no \\
\hline NM_003263 & TLRI & 91 & membrane & I sp, I tms & yes & no \\
\hline NM_032292 & FLJ20203 & 91 & $\mathrm{n} / \mathrm{a}$ & Icc, & yes & no \\
\hline NM_014I49 & HSPC049 & 78 & $\mathrm{n} / \mathrm{a}$ & Icc, & yes & no \\
\hline
\end{tabular}



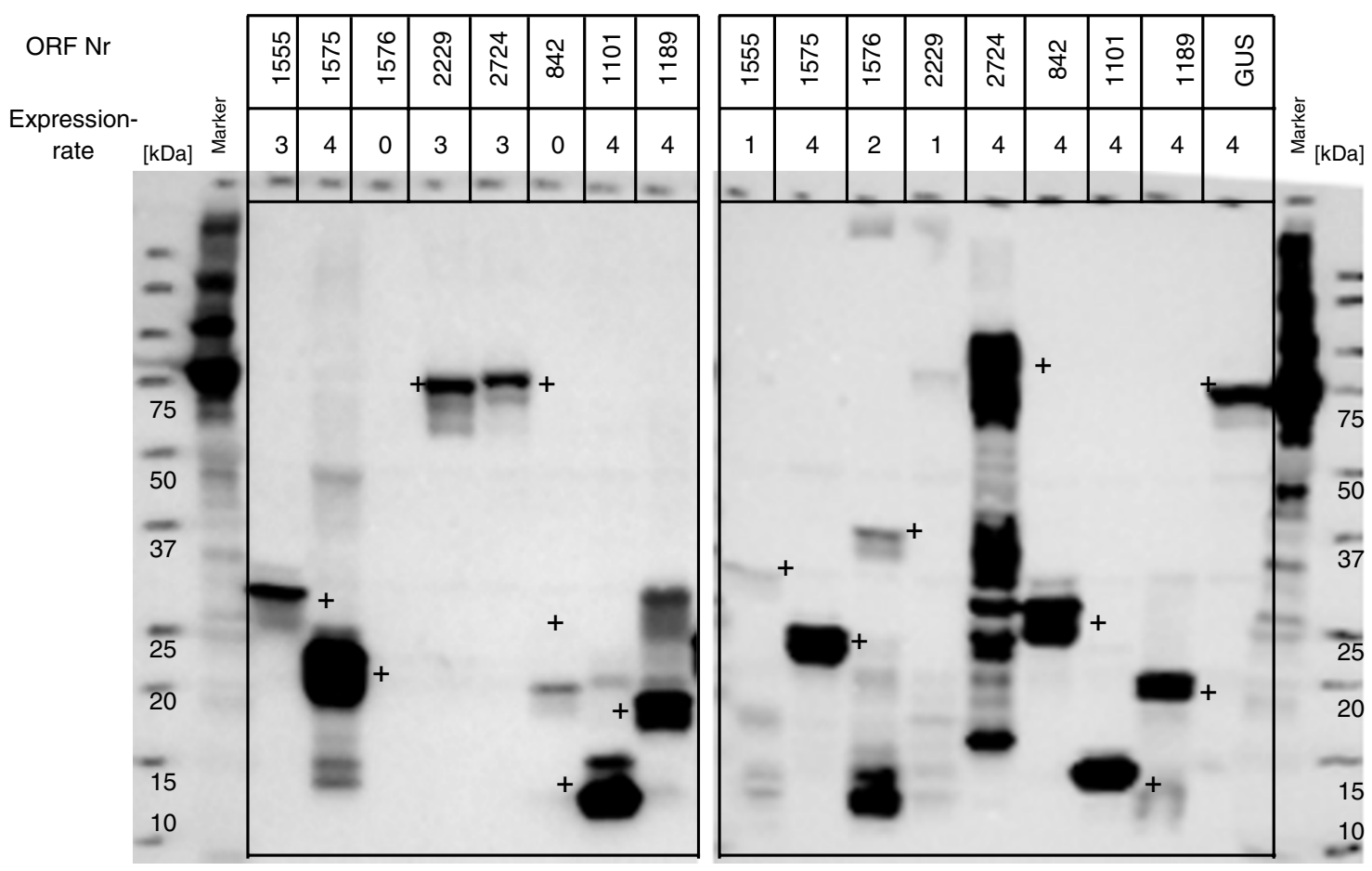

\section{Figure 5}

Wheat germ expression in vitro. Presented are western blots of 8 targets expressed with C-terminal (left) and N-terminal (right) 6xHis-tag. ORF Nr.: clone identifier. GUS: glucuronidase is the positive control. Successful protein expression was defined for values $2-4$ and unsuccessful protein expression for values of 0 and I. Bands of theexpected size are marked with $\mathrm{a}+$.

\section{Influence of tag position on protein expression in vitro} To assess the influence of either $\mathrm{N}$ - or C-terminal tag positions on expression rate, the 87 open reading frames were

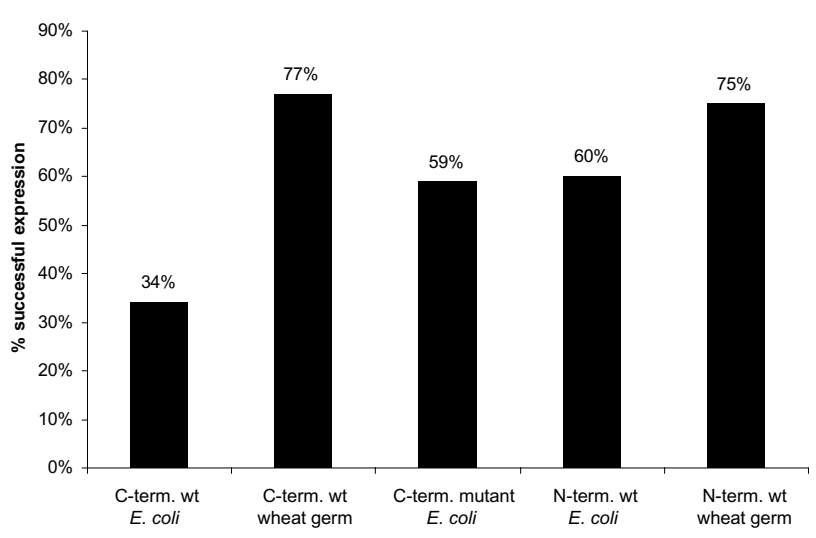

Figure 6

Influence of tag on in vitro expression. We compared 87 targets expressed in E.coli in vitro and in wheat germ. C-term. wt: C-terminal wild type; C-term. mutant: C-terminal mutant; $\mathrm{N}$-term. wt: $\mathrm{N}$-terminal wild type. evaluated in both in vitro expression systems, E. coli and wheat germ (Figure 6). In the E. coli in vitro system, protein expressions using optimised PCR products were evaluated. Here, $52(60 \%) \mathrm{N}$-terminal tagged wild type PCR products expressed protein compared to only 30 (34\%) with C-terminal wild type PCR products (Figure 4). With the C-terminal mutant product 51 (59\%) proteins were expressed. In the wheat germ system, 65 were expressed using the $\mathrm{N}$-terminal wild type construct $(75 \%)$ and 67 with the C-terminal tag (78\%) (Figure 5).

In summary, 81 out of 87 open reading frames were expressed in both in vitro systems, corresponding to a success rate of $93 \%$. Only 6 ORFs yielded no protein in any of the systems tested (Table 2).

Comparison of solubility of proteins expressed in E. coli in vivo, in vitro and in wheat germ system

For solubility studies, the lysis supernatant of those targets revealing expression was analysed by western blot. 483 proteins, expressed in E. coli in vivo, were tested and 193 proteins were soluble $(40 \%)$. For E. coli expressions in vitro (with original plasmid DNA), 388 were analysed and 185 proteins were soluble (48\%). In the wheat germ sys- 
tem with a C-terminal 6xHis-tag 66 of the 68 (97\%) expressing PCR products showed soluble protein and $95 \%$ with an $\mathrm{N}$-terminal $6 x$ His-tag.

\section{Discussion and conclusion}

With this approach we evaluated the performance of three different protein expression systems in vivo and in vitro with a set of 960 full-length open reading frames. For our investigations of protein synthesis we chose Escherichia coli bacteria as it is one of the most common and easy to use systems. For cell-free in vitro expression, we compared the E. coli with the wheat germ protocol.

First, we analysed the protein expression rate in the two $E$. coli systems (in vitro and in vivo) and found that expression is higher in the in vivo system (66\% compared to $48 \%$ ). Regarding the success rate of both protocols, only $22 \%$ of plasmids yielded no protein.

We then focused on a subset of 87 targets which had yielded no protein in E. coli in vivo. These targets expressed with the cell-free wheat germ and E. coli protocol yielded very different protein expression rates. In wheat germ $86 \%$ of the targets were expressed and in cell-free E. coli only $43 \%$. One of the reasons for unsuccessful in vitro protein expression in E. coli may be the presence of secondary structures in mRNA, which may inhibit translation [19]. To solve this problem, we made use of the ProteoExpert software, which predicts possible sequence-related problems and proposes optimised sequences with potentially reduced unfavourable secondary structures $[18,20]$. Out of the 87 proteins that were not expressed in E. coli in vivo, 37 were expressed in vitro using the wild type sequence. Another set of 37 human proteins could be rescued by sequence optimisation and using linear templates for in vitro expression. Therefore, the overall success rate of in vivo negative clones was $85 \%$. This result clearly demonstrates that sequence optimisation is necessary to improve protein synthesis in the E. coli in vitro system.

Furthermore, we analysed the influence of tag position on protein expression rate. We found no difference between $\mathrm{C}$ - or N-terminal tag in the wheat germ system. However, considering the cell-free E. coli system, 60\% successful expression was obtained with the $\mathrm{N}$-terminal wild type PCR product in contrast to only $34 \%$ with the C-terminal one. In this context it is important to realize that this $60 \%$ expression with the $\mathrm{N}$-terminal tag matches with the $59 \%$ obtained with the C-terminal mutant. Obviously, modifying the sequence by adding a sequence optimised peptide tag also avoids expression problems associated with the inition of translation.

After analysis of the 87 optimised expressions in vitro, 6 samples remained that were not expressed in vivo or in vitro (Table 2). This corresponds to a protein expression success rate of $93 \%$. Regarding those proteins, which could not be expressed in either system, it is striking that the molecular mass of all of these targets is higher than 63 $\mathrm{kDa}$ with an average molecular weight of $91 \mathrm{kDa}$. Two membrane associated proteins belong to the unsuccessful targets: PNPLA8 (89 kDa) and SLC40A1 (63 kDa), the latter with more than 10 transmembrane domains. Furthermore, a DNA binding protein KIF22 (74 kDa) of the Kinesin family, involved in spindle formation, ELAC2 (93 $\mathrm{kDa}$ ) an endonuclease, TTLL5 (92 kDa), a tubulin tyrosin ligase-like protein and KIAA1287 (135 kDa), a hypothetical protein with one transmembrane domain, are among the non-expressing targets. At this point it is unclear whether these human proteins are functionally expressed in any of the systems. Therefore, we can not speculate about the interference between the protein function and the different expression systems.

However, 6 proteins with an average molecular weight of $72 \mathrm{kDa}$ were expressed in E. coli in vitro but not in wheat germ. Among these proteins is HMOX2 which belongs to the heme oxygenase family, an iron-containing protein with one transmembrane domain. As reported recently, iron-containing proteins require supplemented iron sources which were not added in this case [21]. Further proteins are two with transmembrane domains (MLSTD1 with two transmembrane domains and TLR1 with one). The three proteins RACGAP1, FLJ20203 and HSPC049 each contain one coiled coil domain and have molecular weights higher than $70 \mathrm{kDa}$. Obviously, the expression of proteins with molecular weights higher than $70 \mathrm{kDa}$ are critical for the wheat germ system [22].

Ten proteins with an average molecular weight of $45 \mathrm{kDa}$ also remain which were expressed in wheat germ but not in E. coli in vitro. An explanation for this can not be found in the structural domains, because a coiled coil and one transmembrane domain were not a hindrance for expression of the proteins mentioned before. Also the molecular weight is not the problem. Regarding the function of these proteins, SUPT7L (transcription regulation factor), EIF2S2 (translation initiation factor) and RPL23A (rRNA binding protein) are proteins which interfere with DNA or RNA. It seems that those proteins are likely to have negative effects on their recombinant expression, when functional active in E.coli cells. Also proteins influencing the cell cycle like DAP (involved in cell death), KIAA1142 (has a kinase motif), PAK4 (kinase, involved in the JNK pathway), GPSM2 (a signalling modulator) and OS-9 (influences cell growth viability) seem to hamper recombinant protein expression.

Based on western blotting analyses, the protein yield in wheat germ was higher compared to expressions in the $E$. 
coli in vitro. This may be due to the fact that the in vitro $E$. coli expression system is a batch method for protein expression, whereas the wheat germ system is based on a two-compartment system. The two chambers are separated by a semi-permeable membrane which concentrates the expressed protein in the $50 \mu$ reaction chamber, but lets compounds required for protein synthesis such as substrates and energy components pass through into the larger feeding chamber. At the same time, potentially inhibitory by-products are diluted via diffusion across the membrane. The wheat germ system showed the highest rate of success compared to expression in E. coli in vitro or in vivo. Thus, for in vitro protein expression, specifically for toxic proteins which can not be expressed in bacteria, the wheat germ system is the method of choice.

Comparing protein solubility in E. coli bacteria and the cell-free E. coli and wheat germ systems, we found that the wheat germ system produces the highest solubility rate $(97 \%)$. This was also reported previously [22]. It should be mentioned that our experimental procedure does not exclude the formation of protein aggregates. Moreover, the data show that the proteins expressed in vitro are more likely to be soluble than those expressed in vivo. However, even though the E. coli in vivo expressions showed, in a first approach, a higher success rate than in vitro, the in vitro system does have advantages. Protein expression is very fast and can be accomplished within a few hours. The expression of toxic gene products allows proteins to be expressed, which are impossible to express in bacteria. Also the use of PCR products is possible, and no clones are necessary for protein expression. However, linear DNA needs to be protected during the in vitro reactions to suppress nuclease activity. In addition, proteins are also more likely to be soluble when expressed in any of the in vitro systems used compared to expression in bacteria.

In summary, we have demonstrated that cell-free protein expression leads to the desired full-length protein with an overall success rate of up to $93 \%$. In our study, wheat germ expression using a two compartment system is the method of choice as it shows high solubility and high protein yield.

\section{Methods}

\section{Expression-vector construction}

The genes used in this study are available from the RZPD full-ORF clone collection. Entry clones containing the genes of interest were generated by utilising the Gateway ${ }^{\infty}$ Cloning technology (Invitrogen). All entry clones were fully sequenced in order to verify the insert within pDONR201. From the entry clone, the ORF was subcloned to a Gateway ${ }^{\oplus}$ destination vector (pDEST17-D18, a modification of pDEST17, Invitrogen) creating an expression clone (LR reaction), which was then transformed into DH10B bacteria. Plasmid DNA of individual clones was used for transformation of BL21 (DE3) pLysS bacteria and for protein expression in vivo as well as for protein expression in the cell-free E. coli system. The pDEST17D18 http://www.rzpd.de destination vector was used to express selected recombinant proteins controlled by the T7 promoter with an N-terminal 6xHis-tag. Identical constructs were used for protein expression in E. coli as well as for expressions in the cell-free E. coli system. All DNA preparations were carried out by a Qiagen Biorobot 9600 using Qiawell 96 Ultra Plasmid Kits (Qiagen).

\section{In vivo protein expression using $\mathrm{E}$. coli bacteria}

Competent BL21 (DE3) pLysS (Novagen) bacteria were transformed with plasmid DNA (pDEST17-D18 containing the gene of interest). The generated expression clones were cultured overnight, diluted 1:50 to a final volume of $3 \mathrm{ml}$, and incubated in 24 -well plates at $30^{\circ} \mathrm{C}$ or $3.5 \mathrm{~h}$ (until the $\mathrm{OD}_{600}$ was $0,4-0,6$ ). Expression was induced with $1 \mathrm{mM}$ IPTG and bacteria cultured for a further 3,5 h at $30^{\circ} \mathrm{C}$. Cells were harvested by centrifugation. A $5 \mu \mathrm{l}$ aliquot of cell-pellet was removed and added to $45 \mu \mathrm{l}$ of SDS sample buffer. $10 \mu \mathrm{l}$ of the sample were then loaded onto a gel for western blotting analysis. An aliquot of the original sample was also saved for analysis of protein solubility.

\section{In vitro protein expression (E. coli) using vector DNA}

In vitro protein expression was carried out using pDEST17D18 plasmid DNA containing the ORF of interest. A cellfree batch expression system (RTS 100 E. coli HY kit, Roche Diagnostics) was utilised and $50 \mu \mathrm{l}$ reactions were prepared according to the manufacturer's instructions. In brief, the samples were incubated at $30^{\circ} \mathrm{C}$ for 4 hours in a thermal cycler. Green fluorescent protein was expressed as control protein. Following incubation, a $5 \mu$ l aliquot was removed and added to $45 \mu \mathrm{l}$ of SDS sample buffer. $10 \mu \mathrm{l}$ of sample were then loaded onto a gel for Western blotting analysis. An aliquot of the original sample was also saved for analysis of protein solubility.

\section{In vitro protein expression (E. coli) using optimised linear PCR products}

Three PCR products were created for each ORF, a C-terminal wild type, a C-terminal mutant and a $\mathrm{N}$-terminal wild type product (Figure 2). Sequence-verified templates were applied for the amplification of PCR products with the Linear Template Generation Set (LTGS, Roche Diagnostics). For the C-terminal mutant template, silent mutations as proposed by ProteoExpert http://www.proteoexpert.com were introduced at the N-terminus of the sequence. PCR was performed using partially matching primers along the first 15 to 20 nucleotides of each ORF. One gene-specific sense primer containing silent mutations, one gene-specific anti-sense and one wild type primer were used to pro- 
duce the first PCR product. Different primers were applied depending on whether a C- or a $\mathrm{N}$-terminal 6xHis-tag was desired. The PCR products were checked on agarose gels, and the second amplification step was carried out according to the supplier's instructions. As positive control protein, green fluorescent protein was expressed. Prior to in vitro expression, all products were verified for correct size and purity. In vitro expression was carried out according to instructions and SDS samples prepared.

\section{In vitro protein expression (wheat germ) using linear PCR products}

Specific PCR products were generated to achieve translation in wheat germ lysate. The first wild type PCR product generated for optimisation in the E. coli in vitro system was utilised to produce a second PCR product for the wheat germ system. Linear templates with a T7 promoter and a Kozak sequence were generated for protein expression in wheat germ lysate. In contrast to PCR products created for the E. coli in vitro system, these products did not contain silent mutations. The first PCR products were made using gene-specific primer pairs and the second amplification step was carried out by the RTS Wheat Germ LTGS kit (Roche Diagnostics) according to instructions. The PCR products were again checked for correct size and purity. Proteins were expressed using the RTS 100 Wheat Germ CECF kit (Roche Diagnostics, positive control: glucuronidase) and contained either a C- or an N-terminal 6xHistag. Samples $(50 \mu \mathrm{l})$ were incubated at $24^{\circ} \mathrm{C}, 900 \mathrm{rpm}$ for $24 \mathrm{~h}$ (ProteoMaster Instrument, Roche Diagnostics), SDS samples prepared for western blotting and an aliquot saved for analysis of protein solubility.

\section{Analysis of protein solubility}

An aliquot of the induced bacterial culture was mixed with a lysis reagent (Pop Culture Reagent, Novagen) and $0.1 \%$ Tween 20 and incubated for $10 \mathrm{~min}$ at room temperature. The sample was centrifuged at $10000 \mathrm{~g}$ for $20 \mathrm{~min}$, the supernatant and the pellet were separated and SDS samples prepared for western blotting analysis. For the in vitro systems, samples were centrifuged directly and the pellet and supernatant separated. Results were expressed as values ranging from 0 (no protein detectable in the supernatant) to 4 (the protein band in the supernatant is stronger than in the pellet). Values of 0 to 1 were defined as insoluble and values of 2 to 4 as soluble protein. Values correspond to: $4>70 \%$; $3>40 \%$; $2>10 \%$; $1<10 \%$ solubility; 0 = unsoluble.

\section{Western blotting}

Western blotting was performed with the Criterion System (BioRad) and 10-20\% gradient pre-cast gels. Samples $(10 \mu \mathrm{l})$ were heated at $95^{\circ} \mathrm{C}$ for $5 \mathrm{~min}$ and loaded onto the gel, which was run at $200 \mathrm{~V}, 400 \mathrm{~mA}$ for $1 \mathrm{~h}$. Following electrophoresis, gels were blotted onto PVDF membranes
(Hybond P, Amersham Pharmacia) at $100 \mathrm{~V}, 1000 \mathrm{~mA}$ for $1 \mathrm{~h}$ and protein transfer checked by briefly immersing the membrane in Ponceau S solution (Sigma). Membranes were thoroughly washed in TBST ( $2 \mathrm{mM}$ Tris/HCl, $\mathrm{pH} 7.6$; $13.7 \mathrm{mM} \mathrm{NaCl}$ and $0.1 \%(\mathrm{v} / \mathrm{v})$ Tween 20) and then blocked for $1 \mathrm{~h}$ in $5 \%$ (w/v) non-fat milk/TBST. Following another $3 \times 15 \mathrm{~min}$ washes in TBST, membranes were incubated with the anti-His mouse antibody (Qiagen, $1: 2000$ in $3 \%(\mathrm{w} / \mathrm{v})$ bovine serum albumin/TBST) overnight at $4^{\circ} \mathrm{C}$. Following incubation with the secondary antibody (Anti-mouse IgG HRP, Southern Biotech) for 1 $h$, membranes were washed three times in TBST and developed with ChemiGlow ${ }^{\circledR}$ (Alpha Innotech) chemiluminescent substrate for $5 \mathrm{~min}$. Images were obtained using a CCD camera system (ChemiImager 5500, Alpha Innotech). Protein bands on western blots were assigned values from 0 (no expression) to 4 (very strong band). Successful protein expression was defined for values of 2 to 4 and unsuccessful expression for values of 0 and 1 . The ratings reflect the relative amount of human fusion protein compared to the reference protein (positive control). $4 \geq$ reference protein; $3 \geq 50 \%$ of r. p.; $2 \geq 10 \%$ of r. p.; no expression $<1<10 \%$ of r.p.; $0=$ no expression.

\section{Authors' contributions}

CL coordinated the experiments and helped to draft the manuscript. BG drafted the manuscript. NW and TS performed the experiments. LE built the database. JL provided plasmids. BK organised funding and helped to draft the manuscript and coordinated the study. All authors read and approved the final manuscript.

\section{Acknowledgements}

This work was supported by Roche Diagnostics $\mathrm{GmbH}$. We thank Andrea Graentzdoerffer, Cordula Nemetz, Kairat Madin, Manfred Watzele and Bernd Buchberger for advice and helpful discussions.

\section{References}

I. Brenner SE: A tour of structural genomics. Nature Reviews Genetics 200I, 2(10):80I-809.

2. Wiemann S, Bechtel S, Bannasch D, Pepperkok R, Poustka A: The German cDNA network: cDNAs, functional genomics and proteomics. J Struct Funct Genomics 2003, 4(2-3):87-96.

3. Imanishi T, Itoh T, Suzuki Y, O'Donovan C, Fukuchi S, Koyanagi KO, Barrero RA, Tamura T, Yamaguchi-Kabata Y, Tanino M, et al.: Integrative annotation of 21,037 human genes validated by fulllength cDNA clones. Plos Biology 2004, 2(6):856-875.

4. Bussow K, Scheich C, Sievert V, Harttig U, Schultz J, Simon B, Bork P, Lehrach $\mathrm{H}$, Heinemann $\mathrm{U}$ : Structural genomics of human proteins - target selection and generation of a public catalogue of expression clones. Microbial Cell Factories 2005, 4:.

5. Hanning G, Makrides S: Strategies for optimising heterologous protein expression in Escherchia coli. TIBTECH 1998, 16:54-60.

6. Braun $\mathrm{P}, \mathrm{Hu} \mathrm{YH}$, Shen BH, Halleck A, Koundinya M, Harlow E, LaBaer $\mathrm{J}$ : Proteome-scale purification of human proteins from bacteria. Proceedings of the National Academy of Sciences of the United States of America 2002, 99(5):2654-2659.

7. Baneyx F: Recombinant protein expression in Escherichia coli. Current Opinion in Biotechnology 1999, 10(5):4 I I-42 I.

8. Madin K, Sawasaki T, Ogasawara T, Endo Y: A highly efficient and robust cell-free protein synthesis system prepared from wheat embryos: plants apparently contain a suicide system 
directed at ribosomes. Proc Natl Acad Sci USA 2000, 97(2):559-564.

9. Sawasaki T, Ogasawara T, Morishita R, Endo Y: A cell-free protein synthesis system for high-throughput proteomics. Proc Natl Acad Sci USA 2002, 99(23): I 4652-I4657.

10. Sawasaki T, Seki M, Sinozaki K, Endo Y: High-throughput expression of proteins from cDNAs catalogue from Arabidopsis in wheat germ cell-free protein synthesis system. Tanpakushitsu Kakusan Koso 2002, 47(8 Suppl): I003-I008.

II. Endo Y, Sawasaki T: High-throughput, genome-scale protein production method based on the wheat germ cell-free expression system. Biotechnology Advances 2003, 2 I (8):695-7I 3.

12. Endo Y, Sawasaki T: Cell-free expression systems for eukaryotic protein production. Curr Opin Biotechnol 2006, I 7(4):373-380.

13. Vinarov DA, Lytle BL, Peterson FC, Tyler EM, Volkman BF, Markley JL: Cell-free protein production and labeling protocol for NMR-based structural proteomics. Nature Methods 2004, I(2): |49-I53.

14. Yokoyama S, Hirota H, Kigawa T, Yabuki T, Shlrouzu M, Terada T, Ito Y, Matsuo Y, Kuroda Y, Nishimura Y, et al.: Structural genomics projects in Japan. Nature Structural Biology 2000, 7:943-945.

15. Busso D, Kim R, Kim SH: Expression of soluble recombinant proteins in a cell-free system using a 96-well format. Journal of Biochemical and Biophysical Methods 2003, 55:233-240.

16. Renesto P, Raoult D: From genes to proteins - In vitro expression of rickettsial proteins. Rickettsiology: Present and Future Directions 2003, 990:642-652.

17. Klammt C, Lohr F, Schafer B, Haase W, Dotsch V, Ruterjans H, Glaubitz C, Bernhard F: High level cell-free expression and specific labeling of integral membrane proteins. European Journal of Biochemistry 2004, 27 I(3):568-580.

18. Langlais C, Gernold N, Scheuermann T, Korn B: The Linear Template Generation set: Optimization of Protein expression in the RTS 100 HY. Biochemica 2003, 3:22-25.

19. de Smit M, J vD: Secondary structure of the ribosome binding site determines translational efficiency: a quantitative analysis. Proc Natl Acad Sci USA 1990, 87( 19):7668-7672.

20. Voges D, Watzele M, Nemetz C, Wizemann S, Buchberger B: Analyzing and enhancing mRNA translational efficiency in an Escherichia coli in vitro expression system. Biochemical and Biophysical Research Communications 2004, 3 I 8(2):60 I-6I4.

21. Boyer ME, Wang CW, Swartz JR: Simultaneous expression and maturation of the iron-sulfur protein ferredoxin in a cell-free system. Biotechnology and Bioengineering 2006, 94(I): I 28-I38.

22. Tyler RC, Aceti DJ, Bingman CA, Cornilescu CC, Fox BG, Frederick RO, Jeon WB, Lee MS, Newman CS, Peterson FC, et al.: Comparison of cell-based and cell-free protocols for producing target proteins from the Arabidopsis thaliana genome for structural studies. Proteins-Structure Function and Bioinformatics 2005, 59(3):633-643.

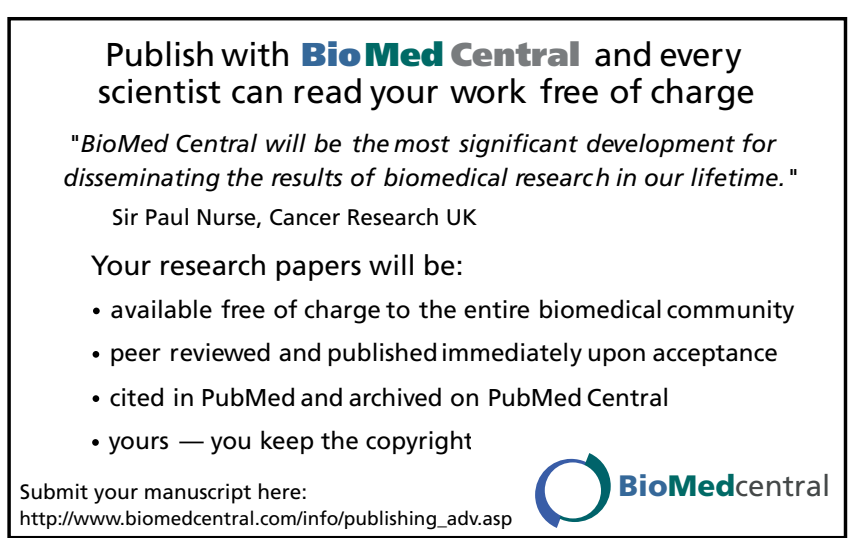

